\title{
DESIGN METHOD OF CIRCULAR WEFT-KNITTED JACQUARD FABRIC BASED ON JACQUARD MODULE
}

\author{
Peixiao Zheng, Gaoming Jiang*, Honglian Cong \\ Engineering Research Center of Knitting Technology, Ministry of Education, Jiangnan University, Wuxi, Jiangsu 214122, China \\ 86-17858505575 \\ * Corresponding author E-mail: jgm@jiangnan.edu.cn
}

\begin{abstract}
:
Recently, there is an increasing interest in design of circular weft jacquard because of the pursuit of fashion and comfort. Aiming at the complexity of the computer-aided design method of the existing circular weft-knitted jacquard fabrics, which is not conducive to the rapid design and intelligible for designers, a design method was proposed to transform pattern notation into knitting diagram efficiently, which was based on knitting rules and its creation as a set of jacquard modules. Knitting characteristics of jacquard fabrics were studied as a precondition. On this basis, the design procedures of jacquard modules were analyzed and illustrated by taking tricolor bird's eye backing jacquard as an example. Jacquard modules with various jacquard effects were designed and stored in a jacquard module database. To mathematically describe pattern notation, knitting diagram, and jacquard module, twodimensional matrixes were established by the method of mathematical modeling, and a corresponding algorithm for the transformation of the pattern to knitting information according to the knitting rules of jacquard modules, which can be applied to ordinary jacquard fabrics was summarized. The project of tricolor circular weft-knitted jacquard with bird's eye in the reverse and four-color air-layer jacquard were taken for instance to verify the models and algorithm. The results obtained show that the approach can efficiently and conveniently realize the designation and machine-knitting of weft-knitted jacquard fabric, which provide a theoretical basis and notation of modeling for the computer-aided design of circular weft-knitted jacquard fabrics.
\end{abstract}

\section{Keywords:}

circular weft knitting; jacquard module; pattern notation; knitting diagram; two-dimensional matrix; computer-aided design

\section{Introduction}

The revolution of an abreast of the times knitting industry is closely linked to advancements in computer science [1, 2]. As an important modern technology, the computer has brought new opportunities for the transformation and upgrading of the knitting industry. Jacquard fabrics is a branch of circular weftknitted products [3], which can be produced by circular weftknitting machines because of the individual needle selection. Because of its ever-changing pattern effects, jacquard fabrics are widely favored, and the market demand is increasing. To meet the efficient, convenient, and innovative requirements of designers, it is a general trend to research computer-aided design (CAD) [4-6] methods and models of circular weftknitted jacquard textiles and develop corresponding auxiliary design platforms. Obviously, the advancement of CAD in the development and production of jacquard fabrics lays a foundation for the digital design and promotes jacquard design to a new start $[7,8]$.

At present, flat-knitting CAD software has developed maturely [9-11]. M1 PLUS system developed by Germany's STOLL and the SDS-ONE APEX system by Japan's SHIMA SEIKI are leading the development trend of flat-knitting CAD technology.
M1 PLUS has multiple views such as logo view, technological view, and textile view. The ways of pattern expression are rich. The function of SDS-ONE APEX is very complete, and the system that can carry on the design of style, pattern, and match color has more advantages in the function of pattern design and simulation. However, both systems are used with specific machines; besides, the design method is complex, and designers need to go through a long period of training. Unfortunately, there is a big gap between the development of circular-knitting CAD and flat-knitting CAD system [12]. Circular-knitting CAD software still stays in the basic design stage, and the design steps are complicated. For the existing circular weft-knitted jacquard design software on the market, to a great extent, the function of the software depends on the corresponding knitting machine. There are three commonly used circular-knitting software are shown in Figure 1. Mayer \& Cie's Paint Knit is relatively mature, but knitting actions of the cylinder and the dial needs to select different color blocks in the pattern notation when designing. Designers who use this software must be clearly aware of the characteristic of knitting machine and jacquard principle; otherwise, wrong knitting actions may damage the machine. The design principle of Terrot's AutoPaint is similar to Paint Knit. AutoPaint choose the knitting actions in different color blocks, which requires 
designers to have a good understanding of the knitting process. The more complex the pattern, the higher the requirement for technicians. The interface of WAC Designer is simple to operate, but it is difficult to meet the processing of complex pattern rapidly, and the expression is not intuitive. WAC Designer just designs the front pattern, and the reverse pattern is controlled by triangles on the machine. According to previous studies, it is important to mention that there are few of theses about the designation and mathematical models of circular weft-knitted jacquard fabrics. Elena and Stefan [1, 13] proposed a method of a programming module to CAD for different knitting pattern structures such as jacquard, intarsia, lace, and others. However, they just introduced the design process of knitting products about knitting structures images but neglected and did not clarify the theory between pattern notation and knitting diagram. Wang et al. [14] suggested a two-dimensional (2D) matrix for describing a jacquard pattern and knitting diagram, and the corresponding algorithm was established. However, there was no uniform method to design for different adverse effects of double-side jacquard fabrics.

The objective of this research is to create a new approach that is more convenient, efficient, and easy to operate for designers to transfer information of pattern notation to knitting actions. This research is based on the study of the knitting characteristics of circular weft-knitted jacquard fabrics. This research introduces an original method called jacquard module as the core of transformation and elaborates the design method of modules. Finally, the mathematical model of pattern notation, knitting diagram, and jacquard module are established and successfully converted by combining a theoretical algorithm with computer programming. The jacquard module database was established and has proven convenient for designers to quickly develop new products and improve enterprise efficiency. This technique is user-friendly and suitable for all designers.

\section{Knitting characteristics}

Circular weft-knitted jacquard stitch is a colored structure formed by combining dyed yarns with loop shape $[15,16]$. Knitting information, which controls knitting needles to form different loop shapes, is generated through the process. Double-sided jacquard fabrics contain double-side ordinary jacquard fabrics and two-side jacquard fabrics (two sides have different patterns). Double-sided ordinary jacquard fabrics are formed by the control of dial's triangles with cylinder's needle selection, and the pattern surface is generally knitted by cylinder's needles. However, two-faced jacquard fabrics are knitted by means of the controlling needle rising both dial and cylinder, and each side can be used as the effected surface. When knitting circular weft jacquard, yarns of any color will be needle-selected knitted in the same color area to form the face pattern and be dial-needle-selected knitted in other color areas to form the back pattern. The backside usually consists of a loop and float according to the jacquard type. According to different knitting actions and fabric effects, circular weft-knitted jacquard fabrics can be divided into single jersey jacquard, horizontal stripe backing jacquard, vertical stripe backing jacquard, bird's eye backing jacquard, air-layer jacquard, etc. Whatever effect a fabric has, the knitting rule is consistent. A knitting rule is divided into two parts, one is needle selector,

\begin{tabular}{|c|c|c|c|c|c|c|c|c|}
\hline \multirow{2}{*}{ I1 } & \multicolumn{3}{|c|}{ Dial } & \multicolumn{5}{|c|}{ Cylinder } \\
\hline & Stitch & Tab Works & \begin{tabular}{l|l}
$s$ & Knit. Sel
\end{tabular} & Knit-Sel & Tab. Wor & & Stitch & Finger \\
\hline $1>$ & 80 & 0 & $=\begin{array}{l}K K \\
106 \\
56\end{array}$ & 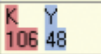 & & 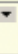 & 120 & \\
\hline 2 & 80 & 0 & $=\frac{1}{24}$ & $\frac{8}{24}$ & 0 & - & 120 & 2 \\
\hline 3 & 80 & 0 & $=\mathbb{R}_{10 \mathrm{~g}}$ & 108 & 0 & - & 120 & 3 \\
\hline 4 & 80 & 0 & $\begin{array}{l}= \\
K \\
100\end{array}$ & $\begin{array}{l} \\
100 \\
\end{array}$ & 0 & - & 120 & 4 \\
\hline
\end{tabular}

(a)

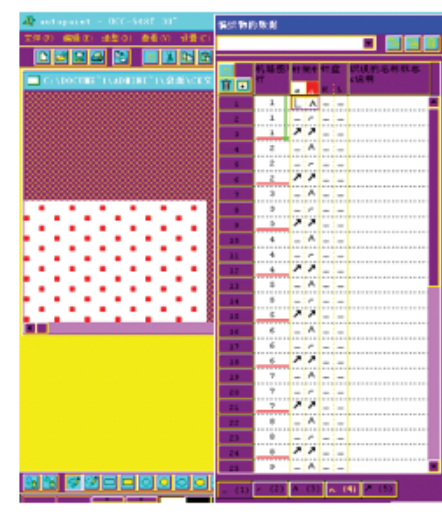

(b)

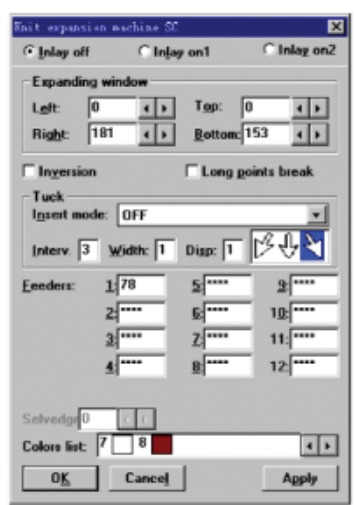

(c)

Figure 1. Jacquard design interface of circular weft-knitted CAD software of (a) Mayer \& Cie's Paint Knit, (b) Terrot's AutoPaint, and (c) WAC Designer

Table 1. Loop list

\begin{tabular}{|c|c|c|}
\hline Loop code $(L c)$ & Icon & Information of knitting action \\
\hline 0 & Floating both in cylinder and dial \\
\hline 1 & Looping in cylinder and floating in dial \\
\hline 2 & Floating in cylinder and looping in dial \\
\hline 3 & Looping both in cylinder and dial \\
\hline
\end{tabular}


which chooses cylinder needles according to pattern, and the other is dial needles, which rise according to the jacquard type and cam. It is necessary to combine these two parts to knit a jacquard fabric with a knitting rule.

To facilitate the following depiction of colored images, the color in the pattern notation is assigned to different color codes. With regard to description of the knitting rule, loop codes $(L C)$ that definite values as $0,1,2,3$ as shown in Table 1, with specific knitting shape and information.

\section{Mathematical modeling}

\subsection{Pattern notation model}

To illustrate a knitting pattern, a raster graphics image [13] with different colors is used and referred to as a pattern notation, as it shown in Figure 2. Pattern notation is mainly used to express the technological face of a fabric in circular weft-knitted jacquard fabrics [17]. Generally, the smallest pattern cycle on the face of a fabric is used as a stitch cycle. Pattern notation is a 2D plane graphic that $2 \mathrm{D}$ data are converted into a $2 \mathrm{D}$ matrix to describe the color information, and the matrix $\boldsymbol{P}$ (Pattern) is shown in equation (1). Let $w$ and $h$ be the width and height of pattern notation, respectively. $P_{j, k}$ represents the color information at the $k^{\text {th }}$ wale and $j^{\text {th }}$ course and is assigned by color code. The specific values of color code are $0,1, \ldots, 255$ :

$$
\boldsymbol{P}=\left[\begin{array}{ccc}
P_{1,1} & \cdots & P_{1, w} \\
\vdots & P_{j, k} & \vdots \\
P_{h, 1} & \cdots & P_{h, w}
\end{array}\right]
$$

After the design of pattern notation, the width $(w)$, height $(h)$, and 2D matrix $\boldsymbol{P}$ can be obtained. The color sequence is an important reference when decomposing the pattern. Therefore, the following one-dimensional matrix $\boldsymbol{C}$ (Color) is used to store different color codes, which are used in matrix $\boldsymbol{P}$ with a certain order. Taking the first pattern grid's information $\boldsymbol{P}_{1,1}$ as the starting point, and the last pattern grid's information $\boldsymbol{P}_{h, w}$ as the end point to traverse the matrix $\boldsymbol{P}$. Finally, different color codes are sequentially stored in the matrix $\boldsymbol{C}$. Let $n$ be the sum of

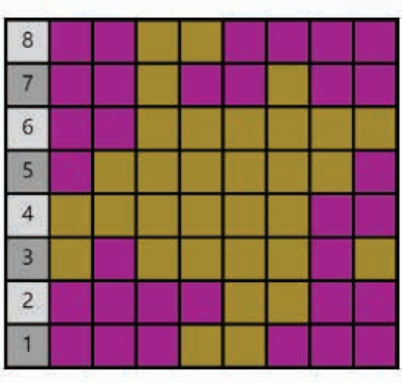

(a)

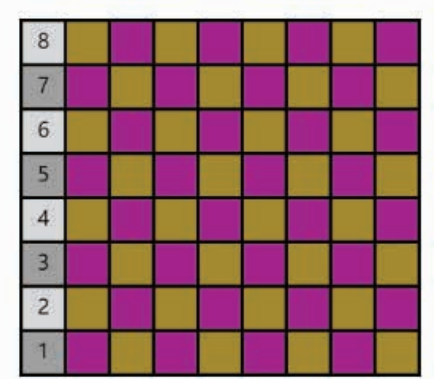

(b)
Figure 2. Pattern notation of (a) front of a pattern and (b) back of a pattern with sesame point different colors in the pattern notation. $C_{i}$ represents the color code of $i^{\text {th }}$ color:

$$
\boldsymbol{C}=\left[\begin{array}{lllll}
C_{1} & \ldots & C_{i} & \ldots & C_{n}
\end{array}\right]
$$

\subsection{Knitting diagram model}

In fact, the information of knitting actions is the real determinant of machine knitting. Knitting diagram illustrated in Figure 3 is used to describe the abstract shape of yarns formed by actions of needles in each knitting row. To display the pattern and knitting information simultaneously, a knitting diagram consists of colorful grids and different stitch icons. In a double-circular weft-knitted jacquard fabric, a group of knitting action consists of actions from a pair of cylinder and dial needles. A jacquard stitch is usually knitted from yarns of different colors or types. Each dyed yarn is knitted at least once in each course to form the front pattern, so it takes $n$ knitting rows to form a single pattern course, where $n$ is the number of different colors in pattern notation. $H$ is defined as the rows of knitting diagram and contains the following mathematical relationship relative to pattern courses $h$ :

$$
H=n \times h
$$

To conveniently convert the information of pattern notation into a knitting diagram, it is necessary to split and copy the pattern information of each course according to knitting rows

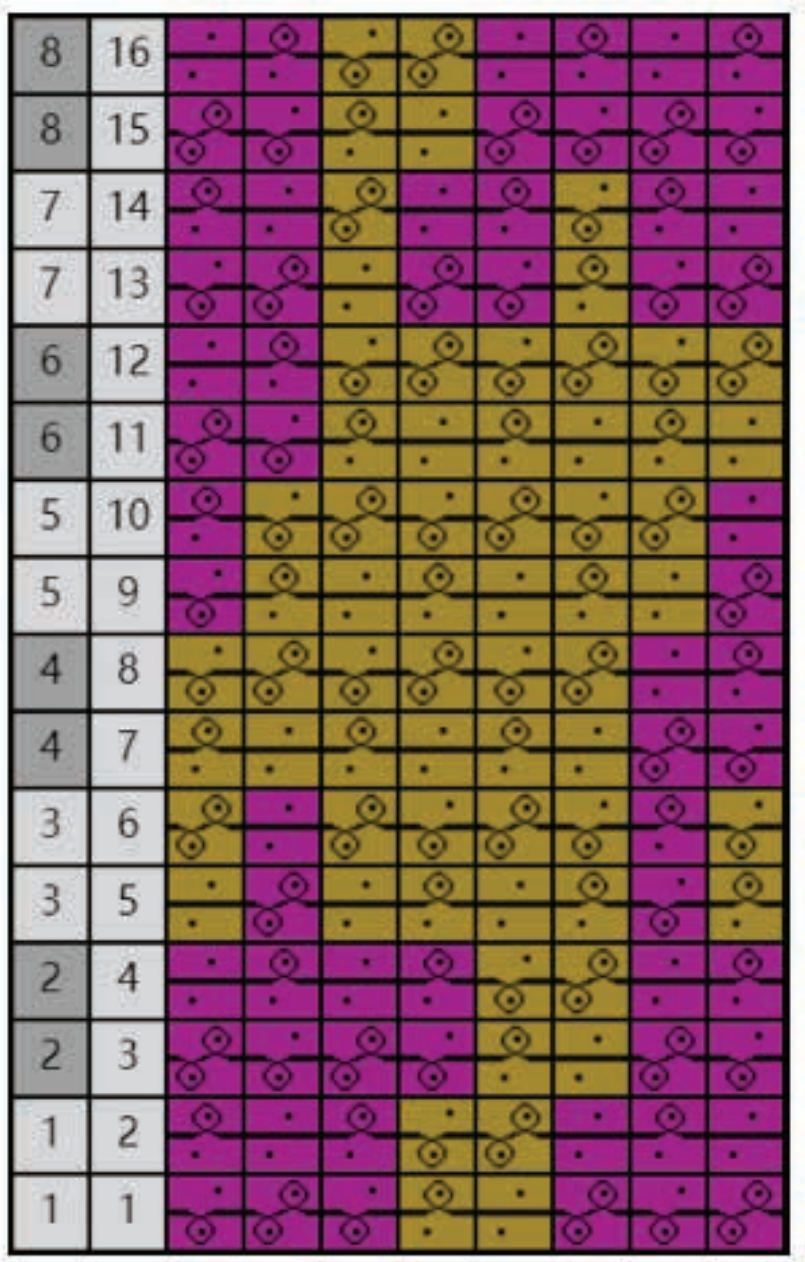

Figure 3. Knitting diagram 


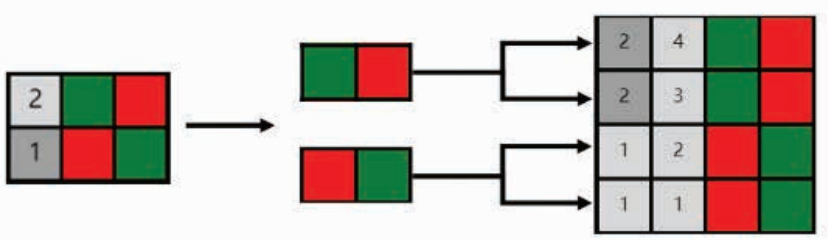

Figure 4. Process from pattern notation to technological notation

n. A 2D matrix $\boldsymbol{T}$ of technological pattern information is defined for storage. The transformational relation between matrix $T$ and $\boldsymbol{P}$ is $T_{j, k}=P_{\operatorname{INT}\left(\frac{j}{n}\right), k \quad \text {, and the process of transformation }}$ from pattern notation to technological notation is illustrated in Figure 4 . To store the generating knitting information, a 2D matrix $K$ is created to describe it. Let $w$ and $H$ be the width and height of knitting diagram, respectively. $\boldsymbol{T}_{j, k}$ represents the color information at the $j^{\text {th }}$ row and $k^{\text {th }}$ wale and is assigned by color code. $\boldsymbol{K}_{j, k}$ represents the knitting information at the $j^{\text {th }}$ row and $k^{\text {th }}$ wale and is assigned by the loop code:

$$
\begin{aligned}
\boldsymbol{T} & =\left[\begin{array}{ccc}
T_{1,1} & \cdots & T_{1, w} \\
\vdots & T_{j, k} & \vdots \\
T_{H, 1} & \cdots & T_{H, w}
\end{array}\right] \\
\boldsymbol{K} & =\left[\begin{array}{ccc}
K_{1,1} & \cdots & K_{1, w} \\
\vdots & K_{j, k} & \vdots \\
K_{H, 1} & \cdots & K_{H, w}
\end{array}\right]
\end{aligned}
$$

\section{Construction of a jacquard module}

\subsection{Analysis of a jacquard module}

A jacquard module is a jacquard needle-selected model with specific jacquard type [18, 19], which is designed according to the structural characteristics of different jacquard types circular jacquard fabrics $[20,21]$. The commonly used jacquard colors are 2 to 4 [14]. There is a two-colored bird's eye backing jacquard module illustrated in Figure 5. According to the knitting rule of yarns, the number of colors in a jacquard module is required to be the same as the number in pattern notation, but the colors do not necessarily correspond. Colors in a jacquard module do not specifically represent actual knitting colors and notation colors, which is only used to distinguish different knitting rows in a pattern course. To distinguish colored yarns knitting in different color regions, the background color of jacquard module is in a staggered arrangement of light and dark gray. The light and dark gray in Figure 5 means that decomposing Region A with knitting rule of wales 1-2 and decomposing Region B with knitting rule of wales 3-4. In the horizontal direction, the number of light gray or dark gray wales is the number of pattern wales for each color; longitudinally, each pair of light gray or dark gray forms a jacquard course, and in this case, $n$ is 2 . When designing, firstly, the smallest courses and wales cycles should be found according to the back effect. The number of smallest rows cycle represents the pattern courses, and the number of smallest columns cycle means the pattern wales of each color in a jacquard module, and they are two important factors need to be identified in the jacquard module. Then, it is necessary to analyze the knitting actions of each color in the

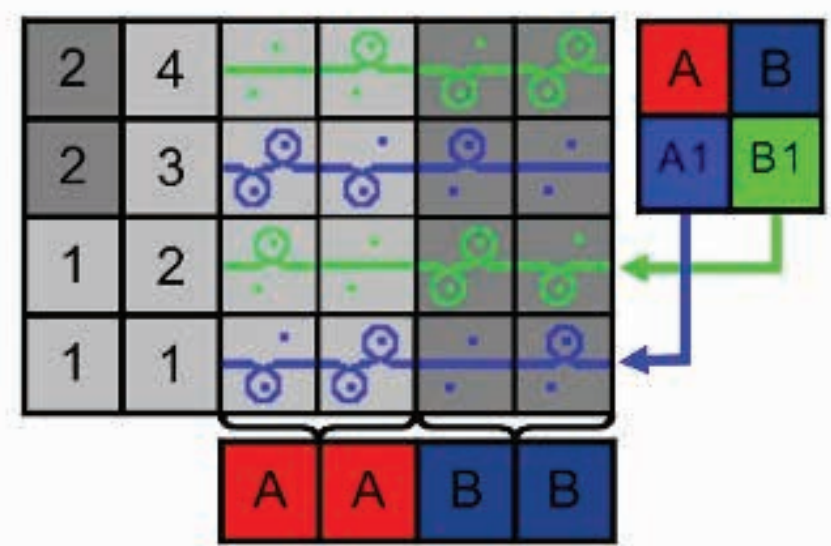

Figure 5.Two-colored bird's eye backing jacquard module and color array

same color area and the rule of loop shape in different color area according to the jacquard type. Finally, these actions are edited into a jacquard module with combining different colors and icons.

\subsection{Design of a jacquard module}

To generate a tricolor bird's eye backing effect shown in Figure 6 (a), there is a certain mathematical relationship between knitting rows' height $(J M H)$ of a designed jacquard module and the minimum cycle pattern's courses (JMh) as equation (6), and the width (JMW) of a jacquard module is calculated by equation (7):

$$
\begin{aligned}
& J M H=n \times J M h \\
& J M W=n \times J M w
\end{aligned}
$$

It can be seen that the number of jacquard colors is three by analyzing Figure 6(a).Furthermore, viewing Figure 6(a) from bottom to top, there are two rows of each color in the minimum cycle that the value of $J M h$ is 2; looking from right to left, $J M w$ is 2 . The reason for looking from right to left is that the direction is from left to right when looked at the front of a fabric. When the fabric is turned over, that is equal to flip the fabric along the $\mathrm{Y}$-axis, the serial number of wales is also reversed. After the discussion above, JMH is 6 and JMW is 6 . The color sequence of pattern is sort by colors $A, B, C$, which, respectively, corresponds to color $\mathrm{A} 1, \mathrm{~B} 1, \mathrm{C} 1$ of the jacquard module. The reverse pattern rule is $A B|C A| B C$ from right to left and bottom to top. As shown in Figure 6(b), the jacquard module can be made according to the needle-outed rule of a tricolor bird's eye backing jacquard fabric. Each pattern course of this kind of fabric has two knitting rows in the front side; however, in the back side, it has three knitting rows. So, the fabric has a characteristic that the ratio of the front to reverse pattern courses is $2: 3$. In the front pattern course of the jacquard module, each color is knitted by needles in the same color region to form front loops, and needles of dial are arranged in $\mathrm{ABC}$ with one interval one needle-outed. As for the reverse side, which is knitted by dial's needles, Yarn A loops at odd needle positions [21] in the first knitting row, and Yarn $\mathrm{B}$ loops at even needle positions in the second row to form the 

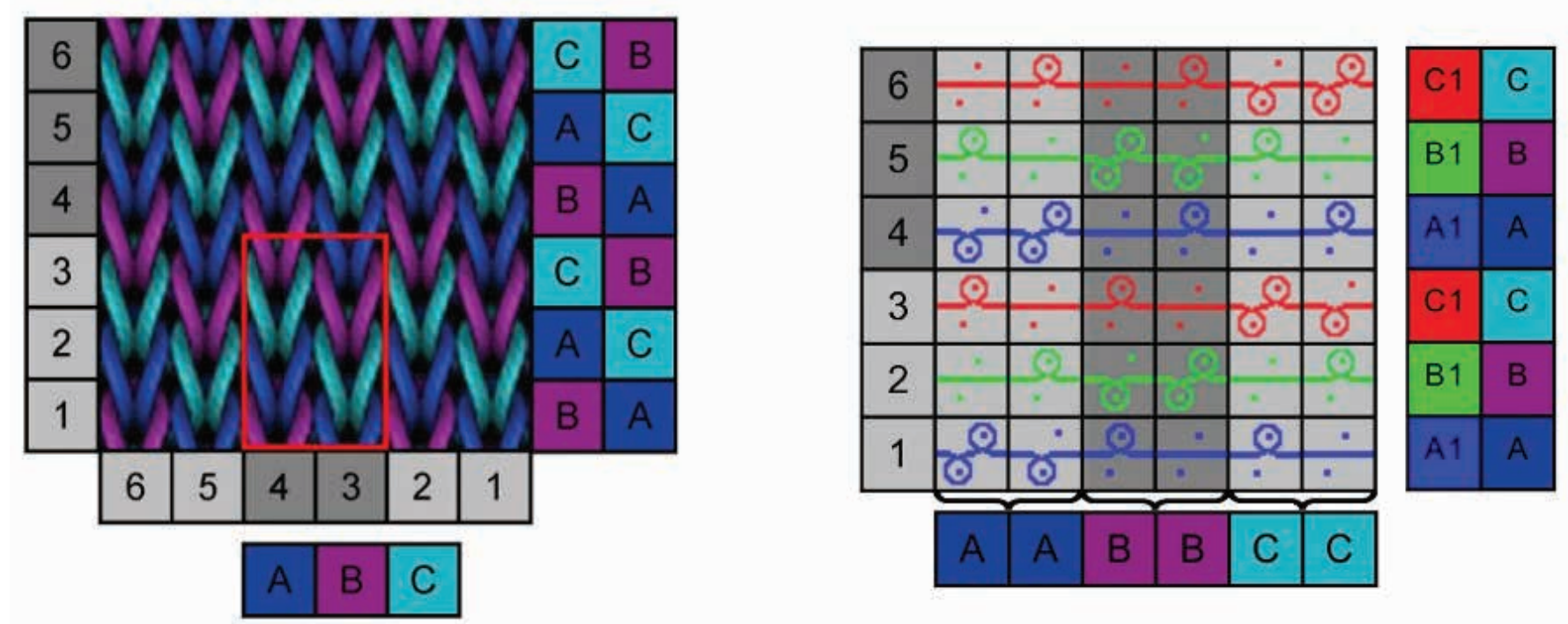

Figure 6. The design of tricolor bird's eye backing jacquard module of (a) tricolor bird's eye effect and (b) jacquard module

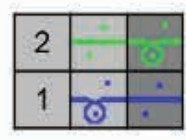

(a)

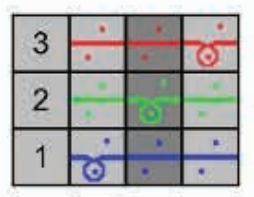

(f)

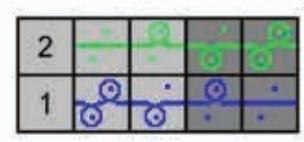

(b)

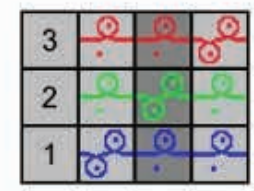

(g)

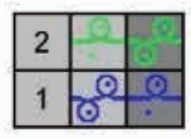

(c)

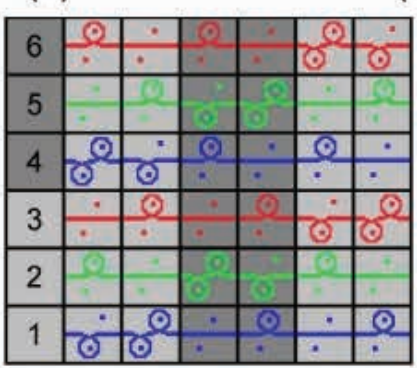

(h)

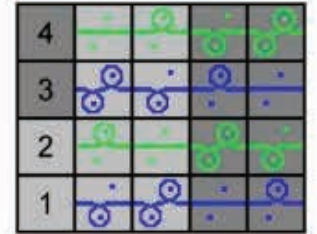

(d)

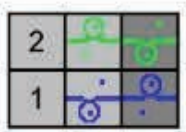

(e)

Figure 7. Jacquard modules of (a) two-color single jersey, (b) two-color vertical stripe backing, (c) two-color horizontal stripe backing, (d) two-color bird's eye backing, (e) two-color air layer, (f) tricolor single jersey, (g) tricolor horizontal stripe backing, (h) tricolor bird's eye backing, (i) tricolor air layer

first course. Yarns $C$ and $A$ are staggered to form the second course. Yarns B and C are staggered to form the third course. There are several common ordinary jacquard modules shown in Figure 7. To conveniently select and make use of various jacquard modules, a jacquard module database is built to store them:

\subsection{Jacquard module modeling}

It is important to mention that the jacquard module mainly sets the knitting actions in each color areas according to the color array. The 2D matrix JM of the jacquard module's knitting information is established as equation (8), $J M_{j, k}$ represents the knitting actions at the $j^{\text {th }}$ row and $k^{\text {th }}$ wale, and the actions are assigned by loop code:

$$
\boldsymbol{J M}=\left[\begin{array}{ccc}
J M_{1,1} & \cdots & J M_{1, J M W} \\
\vdots & J M_{j, k} & \vdots \\
J M_{J M H, 1} & \cdots & J M_{J M H, J M W}
\end{array}\right]
$$

The design of the jacquard module includes its same color area and different color area, and each color area is distinguished with stagger light and dark grays. In the process of converting pattern notation into a knitting diagram, it is necessary to get the knitting actions of the jacquard module according to the color of the pattern notation. To distinguish the knitting rule of each color in a certain color area, matrix $\mathbf{J M}$ is split according to the color areas. A block matrix $J M(i)$ of the jacquard module's knitting information in different color area is established as equation (9), where $i$ is the serial number of the color region: 
$J M(i)=\left[\begin{array}{ccc}J M(i)_{1, J M w \times(i-1)+1} & \cdots & J M(i)_{1, J M w \times i+1} \\ \vdots & J M(i)_{j, k} & \vdots \\ J M(i)_{J M H, J M w \times(i-1)+1} & \cdots & J M(i)_{J M H, J M w \times i+1}\end{array}\right]$

\section{Results and discussion}

\subsection{Pattern decomposition}

After a pattern notation is completed by using drawing tools, the corresponding pattern data can be obtained. It is necessary to design an appropriate jacquard module and get matrix JM and block matrix $J M(i)$. According the conversion flowchart in Figure 8, matrix $\boldsymbol{P}$ can be converted into matrix $\boldsymbol{K}$ according to matrix $\boldsymbol{J} M$. First of all, matrix $\boldsymbol{T}$ is traversed from the starting point of $\boldsymbol{T}_{1,1}$. Secondly, the obtained color information of matrix $\boldsymbol{T}_{j, k}$ is compared with each color code in matrix $\boldsymbol{C}$. If the color is the same as $\boldsymbol{C}_{i}$, the knitting information of matrix $\mathbf{J M}(\boldsymbol{i})_{\mathrm{j}^{\prime} \mathrm{JMH} \mathrm{H}^{\prime} \mathrm{K}^{\prime} \% \mathrm{JMw}}$ is obtained and assigned to $\boldsymbol{K}_{j, k^{*}}$. Finally, matrix $\boldsymbol{K}$ is obtained and saved. If the pattern notation is changed, it is necessary to re-decompose matrix $\boldsymbol{P}$ to obtain a new knitting information matrix $K$.

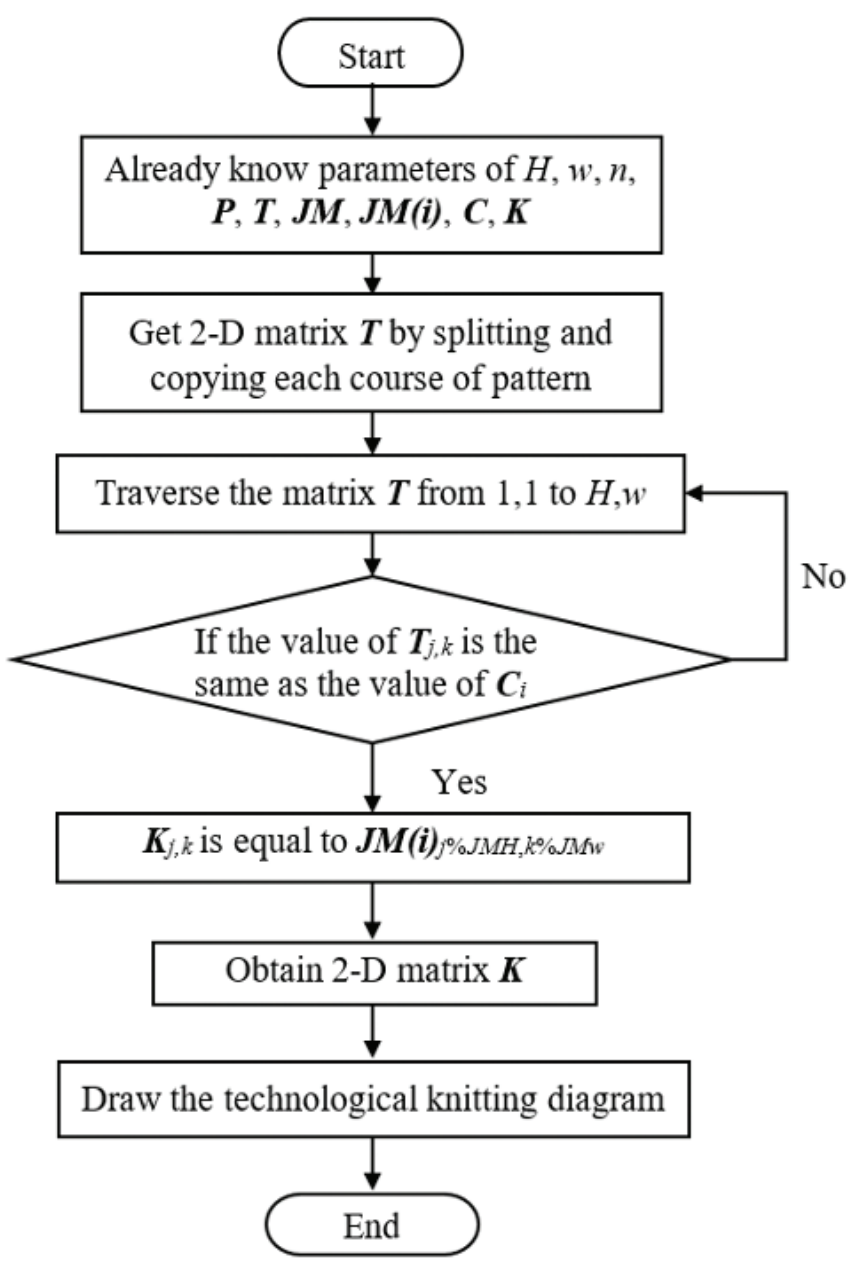

Figure 8. Conversion flowchart of jacquard pattern decomposition
To illustrate the implemental effect of the design method more simply and lucidly, the design of tricolor bird's eye backing jacquard and four-color air-layer jacquard are shown in Figure 9. By means of the above decomposition process, the threecolor bird's eye backing jacquard module (Figure 7(h)) can be used to decompose the intended pattern notation in Figure 9(a) to obtain the knitting diagram, and Figure 9(b) shows the partial knitting diagram. The four-color pattern (Figure 9 (c)) adopts a four-color air-layer jacquard module to get a knitting diagram like Figure 9(d). Only small parts of two knitting diagrams are shown in (b) and (d) to clearly present the knitting actions and color information.

\subsection{Analysis of efficiency}

The circular weft-knitted technology is mature, and types of equipment are abundant. However, the circular-knitting CAD system is always in the basic pattern design stage, lacking a unified and standard design model, and the design difficulty is intricate for non-professionals. This study compares the design efficiency of a jacquard module method, with three commonly used jacquard design software in the market, and the result is shown in Table 2. The evaluation index of design efficiency is the time it takes for three professional designers to design the same pattern using different software. It can be seen that four methods of two-color pattern design are equally simple. For three- and four-color jacquards, both Paint Knit and AutoPaint need to choose the color block or knitting action according to the jacquard effect, which has certain requirements on the theoretical basis of designers. The more the number of colors, the greater the challenge to designers. WAC Designer easily designs the front pattern, but the back pattern is controlled by triangles on the machine. Therefore, it is difficult to design complex patterns and multi-color patterns. Through comparison, it can be found that the method of the jacquard module is relatively simple in the design of multi-color jacquard

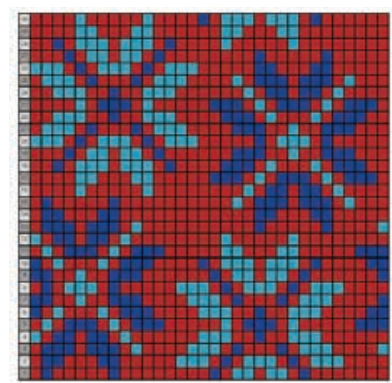

(a)

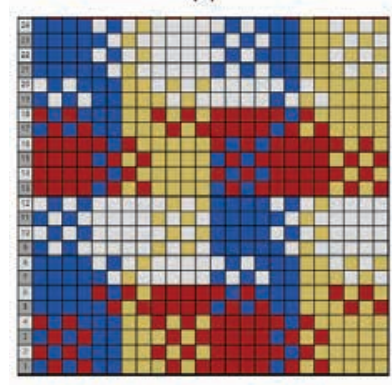

(c)

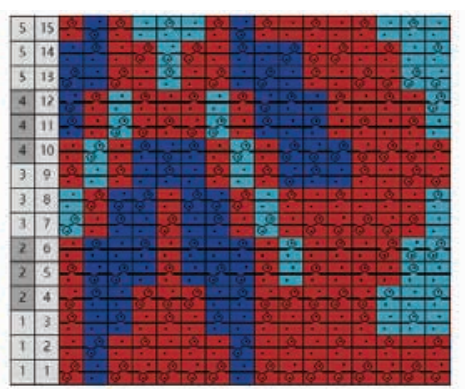

(b)

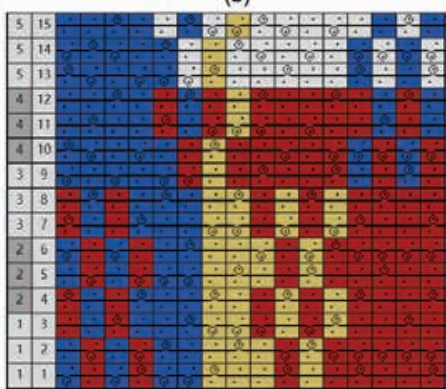

(d)
Figure 9. Jacquard decomposition of (a) tricolor pattern notation, (b) tricolor partial knitting diagram, (c) four-color pattern notation, (d) fourcolor partial knitting diagram 
Table 2. Design efficiency of four circular-knitting jacquard CAD softwares

\begin{tabular}{|c|c|c|c|c|c|}
\hline Pattern & Designers & Paint Knit & AutoPaint & WAC Designer & $\begin{array}{c}\text { Jacquard } \\
\text { module }\end{array}$ \\
\hline \multirow{4}{*}{ Two-color } & $1^{\#}$ & $\sim 4 \mathrm{~m} 23 \mathrm{~s}$ & $\sim 4 \mathrm{~m} 07 \mathrm{~s}$ & $\sim 3 \mathrm{~m} 15 \mathrm{~s}$ & $\sim 3 \mathrm{~m} 50 \mathrm{~s}$ \\
\hline & $2^{\#}$ & $\sim 3 \mathrm{~m} 57 \mathrm{~s}$ & $\sim 3 \mathrm{~m} 52 \mathrm{~s}$ & $\sim 3 \mathrm{~m} 34 \mathrm{~s}$ & $\sim 3 \mathrm{~m} 26 \mathrm{~s}$ \\
\hline & $3^{\#}$ & $\sim 4 \mathrm{~m} 36 \mathrm{~s}$ & $\sim 3 \mathrm{~m} 49 \mathrm{~s}$ & $\sim 3 \mathrm{~m} 21 \mathrm{~s}$ & $\sim 3 \mathrm{~m} 41 \mathrm{~s}$ \\
\hline & AVG & $\sim 4 \mathrm{~m} 19 \mathrm{~s}$ & $\sim 4 \mathrm{~m} 56 \mathrm{~s}$ & $\sim 4 \mathrm{~m} 23 \mathrm{~s}$ & $\sim 3 \mathrm{~m} 39 \mathrm{~s}$ \\
\hline \multirow{4}{*}{ Three-color } & $1^{\#}$ & $\sim 5 \mathrm{~m} 43 \mathrm{~s}$ & $\sim 5 \mathrm{~m} 14 \mathrm{~s}$ & $\sim 4 \mathrm{~m} 47 \mathrm{~s}$ & $\sim 4 \mathrm{~m} 12 \mathrm{~s}$ \\
\hline & $2^{\#}$ & $\sim 6 \mathrm{~m} 09 \mathrm{~s}$ & $\sim 5 \mathrm{~m} 38 \mathrm{~s}$ & $\sim 5 \mathrm{~m} 06 \mathrm{~s}$ & $\sim 3 \mathrm{~m} 48 \mathrm{~s}$ \\
\hline & $3^{\#}$ & $\sim 5 \mathrm{~m} 56 \mathrm{~s}$ & $\sim 6 \mathrm{~m} 07 \mathrm{~s}$ & $\sim 4 \mathrm{~m} 58 \mathrm{~s}$ & $\sim 4 \mathrm{m08s}$ \\
\hline & AVG & $\sim 5 \mathrm{~m} 56 \mathrm{~s}$ & $\sim 5 \mathrm{~m} 40 \mathrm{~s}$ & $\sim 4 \mathrm{~m} 57 \mathrm{~s}$ & $\sim 4 \mathrm{~m} 03 \mathrm{~s}$ \\
\hline \multirow{4}{*}{ Four-color } & $1^{\#}$ & $\sim 12 \mathrm{~m} 05 \mathrm{~s}$ & $\sim 10 \mathrm{~m} 11 \mathrm{~s}$ & $\sim 7 \mathrm{~m} 23 \mathrm{~s}$ & $\sim 5 \mathrm{~m} 34 \mathrm{~s}$ \\
\hline & $2^{\#}$ & $\sim 12 \mathrm{~m} 53 \mathrm{~s}$ & $\sim 11 \mathrm{~m} 06 \mathrm{~s}$ & $\sim 8 \mathrm{m09s}$ & $\sim 5 \mathrm{~m} 57 \mathrm{~s}$ \\
\hline & $3^{\#}$ & $\sim 11 \mathrm{~m} 58 \mathrm{~s}$ & $\sim 10 \mathrm{~m} 43 \mathrm{~s}$ & $\sim 7 \mathrm{~m} 16 \mathrm{~s}$ & $\sim 5 \mathrm{~m} 13 \mathrm{~s}$ \\
\hline & AVG & $\sim 12 \mathrm{~m} 19 \mathrm{~s}$ & $\sim 10 \mathrm{~m} 40 \mathrm{~s}$ & $\sim 7 \mathrm{~m} 36 \mathrm{~s}$ & $\sim 5 \mathrm{~m} 35 \mathrm{~s}$ \\
\hline \multirow{4}{*}{ Multi-color } & $1^{\#}$ & $>17$ & $>15$ & $>12$ & $>6$ \\
\hline & $2^{\#}$ & $>17$ & $>15$ & $>12$ & $>7$ \\
\hline & $3^{\#}$ & $>16$ & $>15$ & $>12$ & $>6$ \\
\hline & AVG & $>17$ & $>15$ & $>12$ & $>6$ \\
\hline
\end{tabular}

fabrics, and the designed jacquard module can be stored in the database for subsequent calls.

\section{Conclusion}

In this work, a fast design method of a jacquard module has been proposed to convert the information of pattern notation to knitting actions for a circular weft-knitted jacquard design. The knitting characteristics of weft-knitted jacquard fabrics are further studied. The design principle of the jacquard module was analyzed, and the design method of ordinary jacquard module was introduced in detail with the design of the threecolor sesame-point jacquard module as an example. The accuracy of the new design method was verified by the design of tricolor and four-color weft jacquard fabrics. Compared with the other three methods, this method was found to be more rapid and effective. In addition, this method is suitable for nonprofessionals, which can reduce the cost of enterprise training. To a large extent, it is convenient for designers to design and develop new products quickly so as to improve enterprise efficiency and benefits. This research provided a reference value for the CAD of circular-knitted jacquard fabrics. However, as the formation and knitting of two-sided weft-knitted jacquard fabrics are complex and man-sized, it is lack of realization of complex patterns in this work that will be reinforced in the future.

\section{Acknowledgments}

The authors acknowledge the financial support from National Science Foundation of China (Grant Numbers 61772238 and 61902150) and Taishan Industry Leading Talents (Grant Number tscy20180224).

\section{References}

[1] Zharieva-Stoyanova E. I., Bozov S. O.(2010). CAD module for knitting patterns design. IEEE International Conference on Automation Quality \& Testing Robotics, 298-304.

[2] Zhang $X$, et al. (2018). A 3D virtual Weft-knitting Engineering learning system based on Unreal Engine 4. Computer Applications in Engineering Education, 26, 2223-36.

[3] Brackenbury, T. (1972). The Geometry of Weft-Knitted Rib Jacquard Fabrics. Journal of the Textile Institute, 63(5), 142-145.

[4] Kaspar, A., Makatura, L., Matusik, W. (2019). Knitting skeletons: a computer-aided design tool for shaping and patterning of knitted garments.

[5] Li, J., Chen, Z. , Ge, M. (2015). Computer-aided design of luminous fiber embroidered fabric and characterization of afterglow performance. Textile Research Journal, 86(11), 1162-1170.

[6] Xiong, Y., Miao, X., Zhang, A., Jiang, G. (2016). Computer simulation for warp-knitted brushed fabric with patterned piles. Textile Research Journal, 86(15), 1659-1667.

[7] Ng FM., Zhou J. (2006). Digital jacquard textile design in a colorless mode. Research Journal of Textile and Apparel, 10(2), 36-42.

[8] Ng M.C.F., Zhou J. (2009). Innovative layered-combination mode for digital jacquard fabric design. Textile Research Journal, 79(8), 737-743. 
[9] Shi, G. F., Zhang, S. L. (2011). Design of pattern preparation system of computerized flat knitting machine based on delphi. Advanced Materials Research, 332-334, 1395-1399.

[10] Jiang, G., Lu, Z., Cong, H., Zhang, A., Dong, Z. (2017). Flat knitting loop deformation simulation based on interlacing point model. Autex Research Journal, 17(4), 361-369.

[11] Lu, Z., Jiang, G. (2017). Rapid simulation of flat knitting loops based on the yarn texture and loop geometrical model. Autex Research Journal, 17(2), 103-110.

[12] Lu Z., Jiang G., Cong H., Yang X. (2016). The Development of the Flat-Knitted Shaped Uppers based on Ergonomics. Autex Research Journal, 16, 67-74.

[13] Zaharieva-Stoyanova E. (2009). Project of CAD Module for Knitting Pattern Design. IASK International Conference E-Activity and Leaning Technologies\&InterTIC, 22-24.

[14] Wang W., Jiang G., Gao Z., Tang M. (2018). Computer aided design system model and algorithm of weft knitted jacquard fabrics. Journal of Textile Research, 39(3), 161166.

[15] Semnani, D., Hadjianfar, M., Aziminia, H., Sheikhzadeh, M. (2014). Jacquard pattern optimizing in weft knitted fabrics via interactive genetic algorithm. Fashion and Textiles, 1(1), 1-9.
[16] Lv T.J., Long H.R. (2015). Feature matching of fancy weft knitted stitch based on modified surf algorithm. Textile Research Journal, 85(7), 751-758.

[17] Wang, J., Yang, B., Huang, B., Jin, Z. (2012). Design and development of polymeric optical fiber jacquard fabric with dynamic pattern display. Textile Research Journal, 82(10), 967-974.

[18] Abounaim MD., Cherif, C. (2012). Flat-knitted innovative three-dimensional spacer fabrics: a competitive solution for lightweight composite applications. Textile Research Journal, 82(3), 288-298.

[19] Sha S., Ma P., Chapman LP., Jiang G., Zhang A. (2017). Three-dimensional modeling and simulation of deformation behavior of fancy weft knitted stitch fabric. Textile Research Journal, 87(14), 1742-1751.

[20] Jin, L., Jiang, G. (2015). Research on the surface of multilayer weft knitted fabrics using computer simulation. International Journal of Clothing ence \& Technology, 27(4), 561-572.

[21] Yukse, C., Kaldor, J.M., James, D.L., Marschner, S. (2012). Stitch meshes for modeling knitted clothing with yarn-level detail. ACM Transactions on Graphics, 31(4CD), 37.1-37.12. 